\title{
Full-wave simulations on ultrashort-pulse reflectometry for helical plasmas
}

H. Hojo, ${ }^{\text {a) }}$ A. Fukuchi, and A. Itakura

Plasma Research Center, University of Tsukuba, Tsukuba 305-8577, Japan

\section{A. Mase}

Art, Science and Technology Center for Cooperative Research, Kyushu University, Kasuga 816-8580, Japan

(Presented on 20 April 2004; published 7 October 2004)

The full-wave simulations on ultrashort-pulse reflectometry for helical plasmas are studied based on the FDTD method in two dimensions. The propagation of an ultrashort-pulse electromagnetic wave is computed in helical plasmas modeled for the Large Helical Device magnetic field configuration. The density-profile reconstruction is performed by the Abel inversion method with the time delay data for the reflected waves from plasma, and it is shown that the reconstructed density profile coincides well with the original profile. (C) 2004 American Institute of Physics.

[DOI: $10.1063 / 1.1790070]$

\section{INTRODUCTION}

Millimeter-wave diagnostics such as reflectometry are receiving growing attention in magnetic fusion research. Recently, detailed measurements on density profile and its fluctuations are required to obtain the better understanding of plasma confinement physics. A type of microwave reflectometry, which is called as ultrashort-pulse reflectometry with use of subcyclic pulses is expected as a near-coming diagnostic. ${ }^{1,2}$ A subcyclic pulse is a ultra-wide-band wave, and it can be considered as a set of monochromatic plane waves with various frequencies corresponding to various cutoff densities, therefore, the ultrashort-pulse reflectometry has a potential of measuring precisely the profiles of plasma density and magnetic field by only a single pulse. The theoretical studies on this ultrashort -pulse reflectometry have been also reported..$^{3-5}$

In this article, we study two-dimensional full-wave simulations on ultrashort-pulse reflectometry for helical plasmas. The simulation scheme is based on the (FDTD) method, ${ }^{5}$ and for a helical magnetic field, an analytic model obtained in Ref. 6 is assumed, which is modeled for the Large Helical Device (LHD) magnetic field configuration. The outline of our simulation model is described in the following section, and in Sec. III, the computational results on the wave propagation of an ultrashort pulse in a LHD plasma are shown, and also the density profile reconstruction by ultrashort-pulse reflectometry with use of the Abel inversion is also demonstrated. It is shown that the ultrashort-pulse reflectometry can work successfully for helical plasmas.

\section{SIMULATION MODEL}

In this section, we describe the two-dimensional simulation modeling for electromagnetic wave propagation developed in Refs. 4 and 5. The basic equations to be solved are

${ }^{a)}$ Electronic mail: Hojo@prc.tsukuba.ac.jp the Maxwell equation for the electromagnetic wave fields, $\boldsymbol{E}$ and $\boldsymbol{B}$, and the equation of motion for the induced current density $\boldsymbol{J}$ as follows:

$$
\begin{aligned}
& \frac{\partial}{\partial t} \boldsymbol{B}=-\nabla \times \boldsymbol{E}, \\
& \frac{\partial}{\partial t} \boldsymbol{E}=c^{2} \nabla \times \boldsymbol{B}-\frac{1}{\varepsilon_{0}} \boldsymbol{J}, \\
& \frac{\partial}{\partial t} \boldsymbol{J}=\varepsilon_{0} \omega_{\mathrm{pe}}^{2} \boldsymbol{E}-\frac{e}{m_{\mathrm{e}}} \boldsymbol{J} \times \boldsymbol{B}_{0},
\end{aligned}
$$

where $c$ is the speed of light, $\omega_{\mathrm{pc}}\left(=\left(e^{2} n / m_{\mathrm{e}} \varepsilon_{0}\right)^{1 / 2}\right)$ the electron plasma frequency, $-e$ the charge of the electron, $m_{\mathrm{e}}$ the electron mass, $n$ the plasma density, $\varepsilon_{0}$ the permittivity of vacuum, and $\boldsymbol{B}_{0}$ is the external magnetic field being corresponding to the helical magnetic field. In the derivation of Eq. (3), we assumed that the current density $\boldsymbol{J}$ is approximated as $\boldsymbol{J}=-e n \boldsymbol{V}_{\mathrm{e}}, \boldsymbol{V}_{\mathrm{e}}$ being the electron flow velocity, as we consider electromagnetic waves in the gigahertz range. The above coupled equations can describe both the ordinary (O) and extraordinary (X) modes in a plasma. When $\boldsymbol{B}$ $=B_{0} \boldsymbol{e}_{z}, \boldsymbol{e}_{z}$ being the unit vector in the $z$-direction, the wave component $E_{z}$ denotes the $\mathrm{O}$ mode with the dispersion relation

$$
\omega^{2}=\omega_{\mathrm{pe}}^{2}+c^{2} k^{2},
$$

where $k$ is the perpendicular wavenumber. On the other hand, $E_{x}$ and $E_{y}$ correspond to the $\mathrm{X}$ mode with the dispersion relation

$$
\left(\frac{k c}{\omega}\right)^{2}=1-\frac{\omega_{\mathrm{pe}}^{2}}{\omega^{2}} \frac{\omega^{2}-\omega_{\mathrm{pe}}^{2}}{\omega^{2}-\omega_{\mathrm{pe}}^{2}-\omega_{\mathrm{ce}}^{2}},
$$

where $\omega_{\mathrm{ce}}\left(=e B_{0} / m_{\mathrm{e}} c\right)$ is the electron cyclotron frequency. The cross polarization scattering between the $\mathrm{O}$ and $\mathrm{X}$ modes due to the magnetic shear is generated from the $\boldsymbol{J} \times \boldsymbol{B}$ term in Eq. (3). 


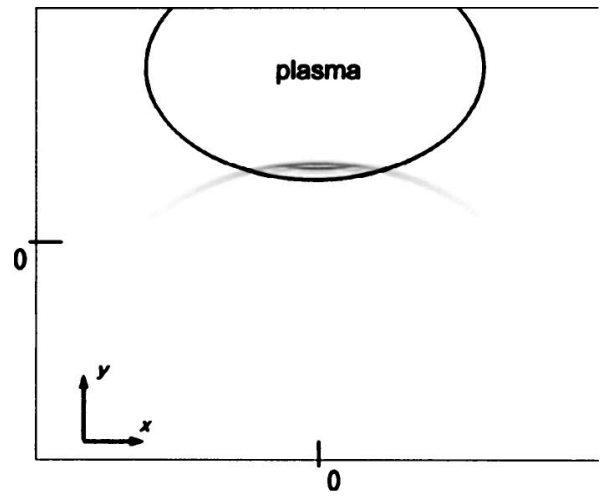

(a)

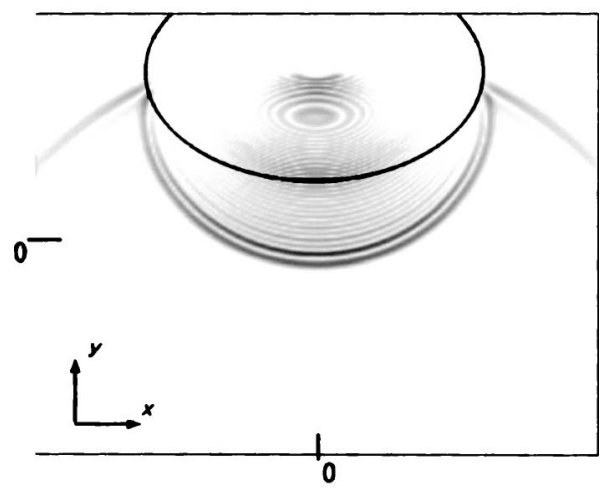

(b)

FIG. 1. (a) The snap shot of an incident pulse $\left(E_{z}\right)$. (b) The snap shot of the reflected wave $\left(E_{z}\right)$.

\section{SIMULATION RESULTS}

In this section, we show the computational results on ultrashort-pulse reflectometry for a helical plasma being modeled as the LHD. We use an analytic expression obtained in Ref. 6 to describe the helical magnetic field $\boldsymbol{B}_{0}$ of the LHD, which is given by

$$
\begin{aligned}
& \boldsymbol{B}_{0}=\nabla \times \boldsymbol{A}+B_{00}\left(\begin{array}{c}
0 \\
0 \\
r_{0} / r
\end{array}\right), \\
& \boldsymbol{A}=\frac{B_{p}}{a}\left(\begin{array}{c}
-\frac{p}{3 r} y^{3}-\frac{p^{3}}{12 r^{3}} y\left(x^{4}+y^{4}\right) \\
-\frac{p}{3 r} x^{3}-\frac{p^{3}}{12 r^{3}} x\left(x^{4}+y^{4}\right) \\
-\frac{x^{2}-y^{2}}{2}\left(1-\frac{r-r_{0}}{4 r}-\frac{p^{4}}{6 r^{4}} x^{2} y^{2}\right)
\end{array}\right),
\end{aligned}
$$

where $r=r_{0}+x \cos (p \phi)-y \sin (p \phi)$, and we here assume that $B_{00}=3 \mathrm{~T}, B_{p}=1 \mathrm{~T}, r_{0}=3.9 \mathrm{~m}, a=0.6 \mathrm{~m}$ and $p=5$. In this case, the toroidal magnetic field is in the $z$-direction, and the poloidal magnetic field is in the $x-y$ plane. We also assume the same parameters as Ref. 6 for the helical magnetic field $\boldsymbol{B}_{0}$. For a density profile, we assume

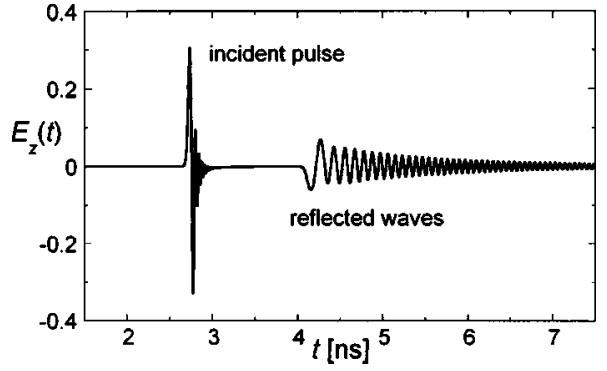

FIG. 2. The wave signals $\left(E_{z}\right)$ as a function of the time at $x=y=0$.

$$
n(x, y)= \begin{cases}n_{0}\left(1-\frac{\psi(x, y)}{\psi_{0}}\right), & \psi \leqslant \psi_{0}, \\ 0, & \psi>\psi_{0}\end{cases}
$$

where $\psi$ is a flux function defined by $\psi=(1-f) x^{2}+(1+f) y^{2}$, $f=r_{0} B_{\mathrm{p}} / a p B_{00}, \psi_{0}$ denotes its value at the vacuum-plasma boundary, which is shown by an ellipse in Figs. 1 and 2, and $n_{0}=5 \times 10^{12} \mathrm{~cm}^{-3}$ is assumed for numerical calculations. We assume a Gaussian pulse with the pulse width of $30 \mathrm{ps}$ (in full width at half maximum) as the incident pulse, which is launched from the lower boundary $\left(y=y_{\min }\right)$ in the $y$-direction. We show the snap shots for the electric field profiles in Figs. 1 and 2. Figure 1 shows the electric wave field $E_{z}$ of the incident pulse, and Fig. 2 shows the reflected wave filed $E_{z}$ from the LHD plasma. We note that $E_{z}$ can be regarded to be approximately O-mode, as the toroidal field is much stronger than the poloidal field. For the reflected wave, we see that $E_{z}$ is almost symmetric with respect to the central line of $x=0$, as is O-mode like. On the other hand, $E_{y}$ and $E_{x}$ are not symmetric with respect to the line of $x=0$, even though the figures for $E_{y}$ and $E_{x}$ are not shown here. It is considered that the asymmetry in $E_{y}$ and $E_{x}$ is due to the strong magnetic shear of the helical field.

We now examine the density profile reconstruction by the measurement of the reflected waves from the cutoff. In Fig. 2, we show the temporal behavior of the electric wave field $E_{z}(t)$ observed at a position of $x=y=0$. In the figure, the earlier large signal shows the incident pulse and the later signal denotes the reflected waves from the LHD plasma. From these reflected wave signals, we can obtain the time delay $\tau(\omega)$ as a function of $\omega$ as shown in Fig. 3, where open circles denotes data computed from the zero crossing method $^{3,4}$ and the solid line shows the curve well fitted by a

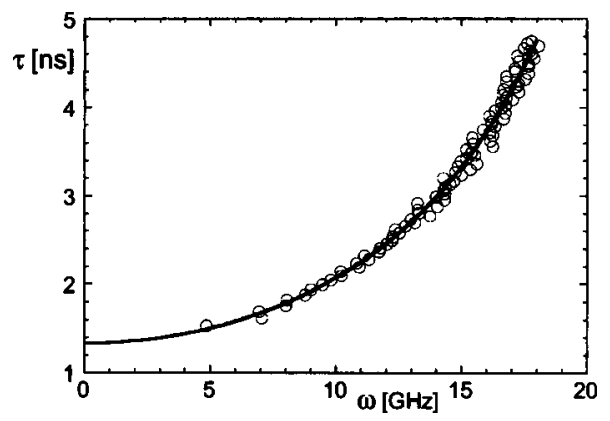

FIG. 3. The time delay $\tau$ of the reflected wave as a function of $\omega$ by the zero-crossing method (open circles) and the nonlinear optimization method (solid line). 


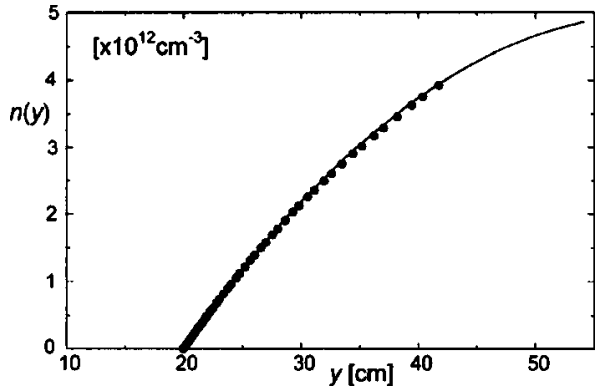

FIG. 4. The reconstructed density profile $n(y)$ at $x=0$ (closed circles). The solid line shows the original density profile.

nonlinear optimization method. ${ }^{7}$ In the optimization method, the time delay $\tau(\omega)$ is approximately expressed as $f(\omega)=c_{0}$ $+c_{1} \omega^{2}+c_{2} \omega^{4}+c_{3} \omega^{6}$ with $c_{0}=1.33$, and its coefficients $c_{j}(j$ $=1 \sim 3$ ) are determined from the minimization condition of the following equation:

$$
I\left(c_{1}, c_{2}, c_{3}\right)=\sum_{i}\left|\tau_{i}-f\left(\omega_{i}\right)\right|^{2},
$$

where the summation is done over all data points. By using the Abel inversion equation ${ }^{3,4}$ defined by

$$
x_{\mathrm{r}}\left(\omega_{\mathrm{pe}}\right)=\int_{0}^{\omega_{\mathrm{pe}}} \mathrm{d} \omega \frac{c \tau(\omega)}{\pi \sqrt{\omega_{\mathrm{pe}}^{2}-\omega^{2}}}
$$

with $\tau(\omega)$ shown in Fig. 3, we can reconstruct the density profile $n(y)$ along the $y$-axis at $x=0$. The reconstructed pro- file is shown in Fig. 4 by closed circles, where the solid line denotes the original density profile. We see that the reconstructed density profile coincides well with the original profile. Finally, as we find that the reflected wave $E_{z}$ is spreading out widely, we might expect the possibility of the multidimensional measurement of the density profile by using a detector array.

\section{ACKNOWLEDGMENTS}

This work was partly performed as a collaborating program at National Institute for Fusion Science, and also was supported by Effective Promotion of Joint Research with Industry, Academia, and Government in Special Coordination Funds for Promoting Science and Technology, MEXT. The simulations were partly performed by the super computer of Science Information Processing Center, University of Tsukuba.

${ }^{1}$ C. W. Domier, N. C. Luhmann Jr., A. E. Chou, W. M. Zhang, and A. J. Romanowski, Rev. Sci. Instrum. 66, 399 (1995).

${ }^{2}$ S. Kubota, T. Onuma, A. Mase, T. Tokuzawa, N. Oyama, A. Itakura, H. Hojo, L. Bruskin, T. Tamano, K. Yatsu, C. W. Domier, and N. C. Luhmann Jr., Jpn. J. Appl. Phys., Part 2 37, L300 (1998).

${ }^{3}$ B. I. Cohen, E. B. Hooper, T. B. Kaiser, E. A. Williams, and C. W. Domier, Phys. Plasmas 6, 1732 (1999).

${ }^{4}$ H. Hojo, R. Kurosawa, and A. Mase, Rev. Sci. Instrum. 70, 983 (1999).

${ }^{5}$ H. Hojo, T. Uruta, A. Fukuchi, and A. Mase, J. Plasma Fusion Res. 78, 387 (2002).

${ }^{6}$ T. Watanabe and H. Akao, J. Plasma Fusion Res. 73, 186 (1997).

${ }^{7}$ L. C.W. Dixon, Nonlinear Optimisation (The English Universities Press, London, 1972). 